\title{
Avaliação dos parâmetros radiográficos do alinhamento sagital e espinopélvico em pacientes com osteoartrite submetidos à artroplastia total do quadril*
}

\section{Evaluation of the Radiographic Parameters of Sagittal and Spinopelvic Alignment in Patients with Osteoarthritis submitted to Total Hip Arthroplasty}

\author{
Vanessa da Costa Sousa ${ }^{10}$ Jamila Alessandra Perini, ${ }^{10}$ Antônio Eulálio Pedrosa Araújo Junior ${ }^{10}$ \\ João Antônio Matheus Guimarães ${ }^{1 \oplus}$ Maria Eugenia Leite Duarte ${ }^{1 \odot}$ Marco Bernardo Cury Fernandes ${ }^{1 \odot}$ \\ ${ }^{1}$ Divisão de Pesquisa, Instituto Nacional de Traumatologia e \\ Ortopedia, Rio de Janeiro, RJ, Brasil \\ 2 Laboratório de Pesquisa de Ciências Farmacêuticas, Unidade de \\ Farmácia, Centro Universitário Estadual da Zona Oeste (UEZO), \\ Endereço para correspondência Vanessa da Costa Sousa, MS, \\ Instituto Nacional de Traumatologia e Ortopedia, Av. Brasil, 500, Caju, \\ Rio de Janeiro, RJ, 20940-070, Brasil \\ (e-mail: vanessamedrj@gmail.com).
} Rio de Janeiro, Brasil

Rev Bras Ortop 2020;55(5):591-596.

\section{Resumo}

Palavras-chave

- alinhamento sagital

- artroplastia de quadril

- osteoartrite

- parâmetros espinopélvicos
Objetivo Avaliar os parâmetros radiográficos do alinhamento sagital e espinopélvico de pacientes com osteoartrite $(\mathrm{OA})$ de quadril submetidos à artroplastia total de quadril (ATQ) primária, com o intuito de definir a abordagem cirúrgica primária em pacientes com doença concomitante na coluna vertebral e na articulação do quadril. Métodos Estudo longitudinal, prospectivo, comparativo, envolvendo 27 pacientes submetidos à ATQ e 43 indivíduos sem OA.

Resultados Foi observada associação entre doença degenerativa no quadril e na coluna nos pacientes com OA. Após a ATQ, os parâmetros radiográficos do ângulo da báscula da bacia, do eixo vertical sagital (EVS) e da razão de C7/DSF (sétima vértebra cervical/distância sacrofemoral) foram semelhantes aos valores dos voluntários sem doença articular. O alinhamento coronal global (ACG), o alinhamento sagital, as inclinações T1 e T9 espinopélvicas (IT1EP e IT9EP), a inclinação sacral (IS), a versão pélvica (VP), e o tipo de pelve e do complexo lombopélvico (CLP) não sofreram alteração depois da ATQ.

Conclusão Dentre os parâmetros do alinhamento sagital e espinopélvico avaliados, o ângulo da báscula da bacia, o EVS e a razão C7/DSF foram corrigidos após a ATQ e podem orientar o cirurgião na tomada de decisão para pacientes com doença concomitante na coluna vertebral e na articulação do quadril. A deformidade da coluna pode ser compensatória às alterações do quadril.

* O presente trabalho foi realizado no Instituto Nacional de Traumatologia e Ortopedia (INTO), Rio de Janeiro, RJ, Brasil.

recebido

18 de Março de 2019

aceito

30 de Outubro de 2019
DOI https://doi.org/

10.1055/s-0040-1701286. ISSN 0102-3616.
Copyright $\odot 2020$ by Sociedade Brasileira License terms de Ortopedia e Traumatologia. Published by Thieme Revinter Publicações Ltda, Rio de Janeiro, Brazil 


Abstract
Keywords
- sagittal alignment
- arthroplasty,
replacement, hip
- osteoarthritis
- spinopelvic
parameters

Objective To evaluate radiographic parameters of sagittal and spinopelvic alignment in patients with hip osteoarthritis (OA) undergoing primary total hip arthroplasty (THA) to define the primary surgical approach in individuals with concomitant spinal and hip joint disease.

Methods Longitudinal, prospective, comparative study with 27 patients undergoing THA and 43 subjects without OA.

Results An association between hip and spine degenerative disease in patients with OA was noted. After THA, radiographic parameters of pelvic tilt angle, sagittal vertical axis (EVS) and seventh cervical vertebra/sacrofemoral distance (C7/DSF) ratio were similar to values from volunteers without joint disease. Global coronal alignment (ACG), sagittal alignment, spinopelvic T1 and T9 tilts (IT1EP and IT9EP), sacral tilt (IS), pelvic version (VP), pelvic type and lumbopelvic complex (CLP) did not change after THA.

Conclusion Among the sagittal and spinopelvic alignment parameters evaluated, the pelvic tilt angle, the EVS, and the C7/DSF ratio were corrected after THA and can guide the surgeon in the decision-making process for patients with concomitant spinal and hip joint disease. Spinal deformity may compensate for hip changes.

\section{Introdução}

A associação entre as doenças na articulação do quadril e as alterações no alinhamento sagital e espinopélvico repercutem diretamente sobre o equilíbrio da postura e sobre o alinhamento da coluna-pelve, contudo os mecanismos responsáveis por esta associação não são totalmente conhecidos. ${ }^{1,2}$ No paciente com osteoartrite ( $\mathrm{OA})$ no quadril, a interação entre a coluna lombossacra e a pelve dificultam o diagnóstico diferencial sobre o local primário relacionado com a dor. ${ }^{3} \mathrm{~A}$ idade é o principal fator de risco para o desenvolvimento da OA, podendo atingir 30\% das pessoas acima de 60 anos. ${ }^{4}$ Por ser uma doença sistêmica comum do envelhecimento, a OA pode acometer simultaneamente a coluna vertebral e o quadril, sendo um desafio no diagnóstico quando o paciente apresenta sintomas e sinais radiológicos de doença degenerativa em ambos os sítios (síndrome coluna-quadril). ${ }^{5-7}$ Além disso, existe um maior risco de complicações após a artroplastia total do quadril (ATQ), resultando em luxação da prótese, necessidade de ATQ de revisão, fratura periprotética e infecção nos pacientes com doença da coluna vertebral. ${ }^{6}$ Dessa forma, torna-se necessário identificar a origem do quadro álgico do paciente, seja ele do quadril ou da coluna, para definir a prioridade do tratamento. Os parâmetros radiográficos do alinhamento sagital e espinopélvico surgem como uma ferramenta importante para aumentar a precisão diagnóstica e auxiliar no direcionamento correto do tratamento. ${ }^{1-14}$ Contudo, não existem dados suficientes na literatura sobre a real contribuição no diagnóstico diferencial entre lesões localizadas na coluna e no quadril. Assim, o objetivo do presente trabalho foi descrever e avaliar os parâmetros radiográficos do alinhamento sagital e espinopélvico de pacientes com OA de quadril, antes e após a cirurgia de ATQ primária, com o intuito de definir se a ATQ deve ser a abordagem primária em pacientes com doença concomitante na coluna vertebral e na articulação do quadril.

\section{Materiais e Métodos}

Foi realizado um estudo longitudinal, prospectivo, comparativo, envolvendo 27 pacientes diagnosticados com OA de quadril, submetidos à ATQ primária, e 43 indivíduos saudáveis, sem doença na articulação do quadril ou na coluna vertebral. 0 protocolo do estudo foi aprovado pelo Comitê de Ética em Pesquisa (58789616.7.0000.5273/2016) e todos os participantes concordaram e assinaram o Termo de Consentimento Livre e Esclarecido.

Os critérios de inclusão dos casos foram: diagnóstico de $\mathrm{OA}$ no quadril, submetidos à cirurgia de ATQ primária entre dezembro de 2016 e setembro de 2017; e de exclusão: acompanhamento radiológico $<80$ dias após a ATQ exames de imagem com qualidade inadequada, história de cirurgia prévia, doenças da infância ou trauma e pacientes com condições neurológicas. O grupo controle teve como critérios de inclusão: idade $>18$ anos, índice de massa corporal (IMC) $<36 \mathrm{~kg} / \mathrm{m}^{2} \mathrm{e}$ ausência de dor ou doença na coluna vertebral ou no quadril; e de exclusão: presença de deformidades, exames de imagem com qualidade inadequada, diagnóstico radiológico de doença no quadril ou na coluna vertebral.

Foram coletadas informações sociodemográficas e clínicas e foram realizadas as radiografias panorâmica em ortostatismo da coluna vertebral nas incidências anteroposterior (AP) e perfil, e na incidência em perfil de coluna lombar-pelvequadril-fêmur proximal na posição ortostática e sentado. Os parâmetros radiográficos (-Fig. 1-2 a 3) foram medidos por dois médicos especialistas, sendo comparados, antes e depois da realização de ATQ e com o grupo saudável. Os marcos de referência dos parâmetros radiográficos foram localizados manualmente por etapas. Na incidência sagital foram identificadas as cabeças femorais, o platô superior da primeira vértebra sacral (S1), o platô superior da primeira vértebra lombar (L1), o platô superior da primeira vértebra torácica (T1) 


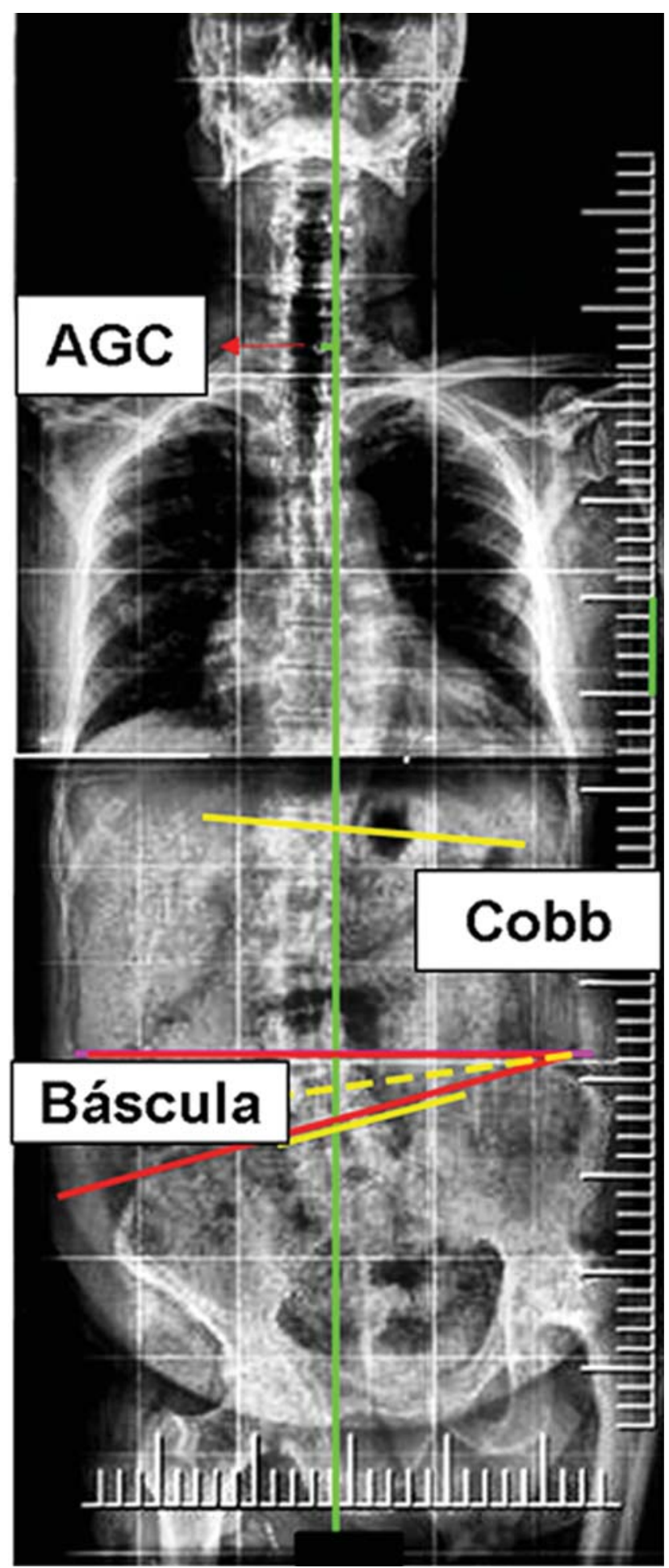

Fig. 1 Parâmetros radiográficos da coluna na incidência anteroposterior. Medidas do alinhamento coronal global (ACG) e do ângulo de Cobb em amarelo e da báscula da bacia em vermelho.

e o platô inferior da segunda vértebra cervical (C2). Na incidência AP foram identificados o ponto médio de S1 e o ponto médio da sétima vértebra cervical (C7). Nos pacientes que apresentavam escoliose, foram identificados os limites das vértebras para cada curvatura (ângulo de Cobb). Nos pacientes que apresentavam báscula de bacia, foram identificadas a asa maior dos ilíacos e a medida manual com a horizontal. A distância sacrofemoral (DSF) foi medida manualmente. Os seguintes parâmetros foram calculados automaticamente pelo programa: EVS, lordose lombar (LL), cifose torácica (CT), inclinações T1 e T9 espinopélvicas (IT1EP e IT9EP), ângulo T1 pélvico (ATP), alinhamento coronal global (ACG) e ângulo de Cobb (na posição ortostática, - Fig. 1 e 2); versão pélvica (VP), incidência pélvica (IP), inclinação sacral (IS) (na posição ortostática e sentada, - Fig. 3). Os tipos de pelve classificados de acordo com o valor da IP foram: $<40^{\circ}$, pelve tipo 1 ; entre $40^{\circ} \mathrm{e}$ $60^{\circ}$, tipo $2 ;>60^{\circ}$, tipo 3.0 CLPera tipo 1 quando seu valor era $\leq$ $40^{\circ}$, e tipo $2 \mathrm{se}>40^{\circ}$ tipo $2 .^{10,11}$ As imagens radiológicas digitais codificadas no padrão DICOM foram visualizadas no programa eFilm Workstation (Merge Healthcare, Chicago, IL, EUA), versão em português 2.0 e foram copiadas para o programa Surgimap Spine (Nemaris Inc., Nova York, NY, EUA), versão 2.2.13.

As variáveis contínuas foram apresentadas como média \pm desvio padrão (DP) e avaliadas utilizando o teste $t$ de Student. Os dados categóricos foram expressos em porcentagem e avaliados pelo teste qui-quadrado de Pearson $\left(X^{2}\right)$ ou pelo teste exato de Fisher. Para avaliar a magnitude de associação dos parâmetros da coluna vertebral com a presença da $\mathrm{OA}$ foram obtidas razões de chance (RC) e seus respectivos intervalos de confiança (IC 95\%).

\section{Resultados}

A média da idade dos voluntários sem doença articular foi de $35,2 \pm 9,4$ anos (18-56 anos) e nos casos de OA foi 58,3 $\pm 11,4$ anos (37-85 anos). Em relação ao gênero e ao IMC, não houve diferença significativa entre os dois grupos $(p<0,05)$.

As principais comorbidades dos pacientes submetidos a ATQ foram hipertensão arterial sistêmica $(n=14)$, obesidade $(n=8)$ e diabetes mellitus $(n=6)$. Em 9 pacientes foi diagnosticada a forma primária da doença e 18 pacientes apresentaram OA secundária, sendo 12 osteonecrose da cabeça femoral. Ambos os lados do quadril foram acometidos na mesma proporção e um paciente era bilateral. O tempo médio de evolução da $\mathrm{OA}$ foi de 8,4 anos (variação entre 3 e 36 anos). 0 tempo médio para a realização da radiografia no pós-operatório foi de 121 dias (variação entre 80 e 124 dias).

Após a ATQ cinco dos seis pacientes com diagnóstico radiológico de escoliose apresentaram melhora das curvas escolióticas, sendo quatro corrigidos (ângulo de $\mathrm{Cobb} \leq 10^{\circ}$ ). Dentre os pacientes com OA, foi diagnosticada espondilolistese em dois casos e colapso de corpo vertebral em três pacientes. Foram observadas alterações degenerativas na coluna vertebral, tais como osteófitos marginais $(n=25)$, redução dos espaços intervertebrais $(n=20)$ e artrose interapofisária $(n=17)$. Foi observada diferença significativa do parâmetro estrutural de altura do corpo vertebral e das alterações degenerativas da coluna entre os casos de $\mathrm{OA}$ e os controles. No grupo dos pacientes com $\mathrm{OA}$, não foi observada associação entre obesidade (IMC $>30 \mathrm{~kg} / \mathrm{m}^{2}$ ) e alterações degenerativas da coluna vertebral.

Não foi observada associação do alinhamento sagital positivo ou negativo com OA de quadril, antes ou depois da ATQ. A 

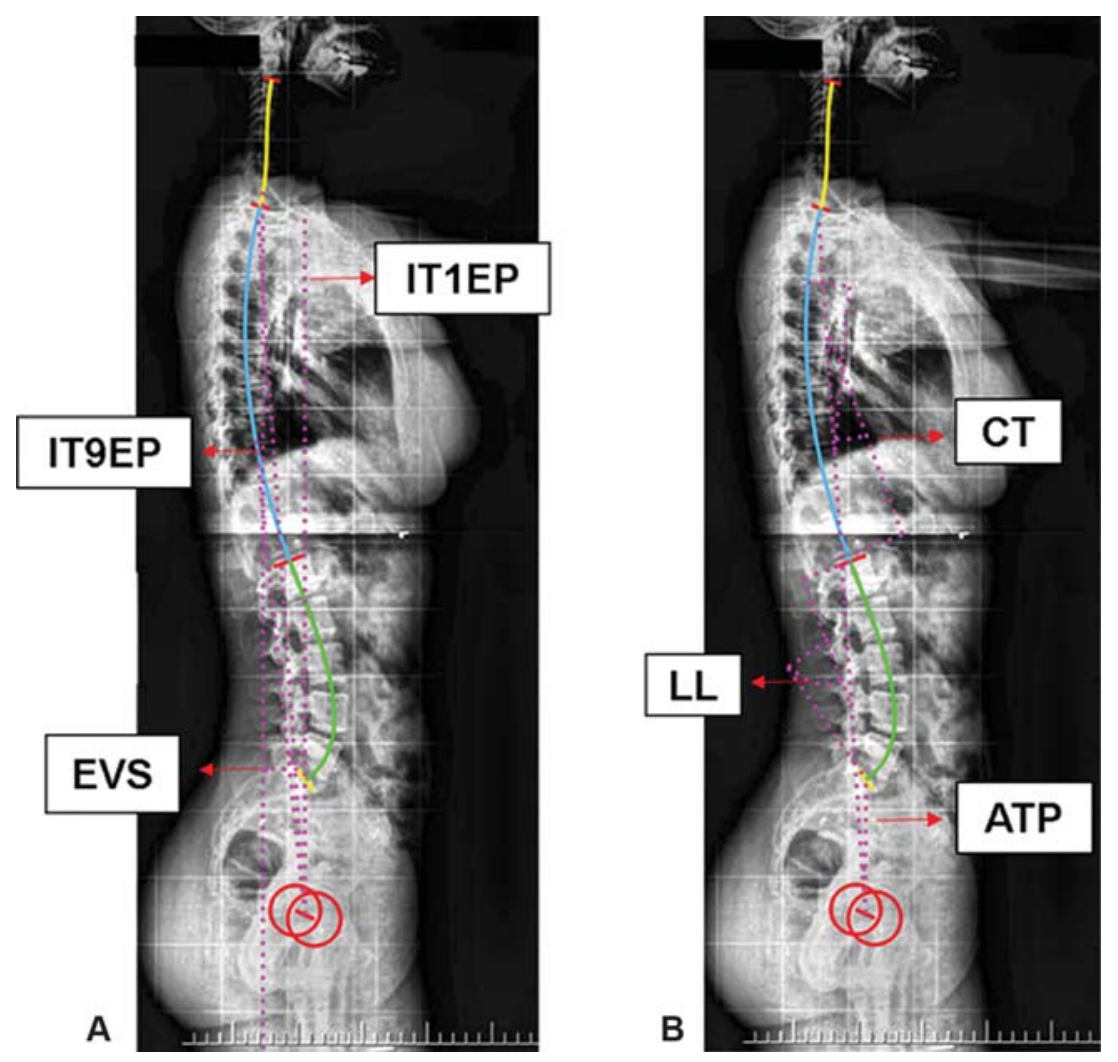

Fig. 2 Parâmetros radiográficos da coluna na incidência em perfil. (A) Eixo vertical sagital (EVS), Inclinação T1 espinopélvica (IT1EP) e inclinação T9 espinopélvica (IT9EP). (B) Cifose Torácica (CT), lordose lombar (LL) e ângulo T1 Pélvico (ATP).
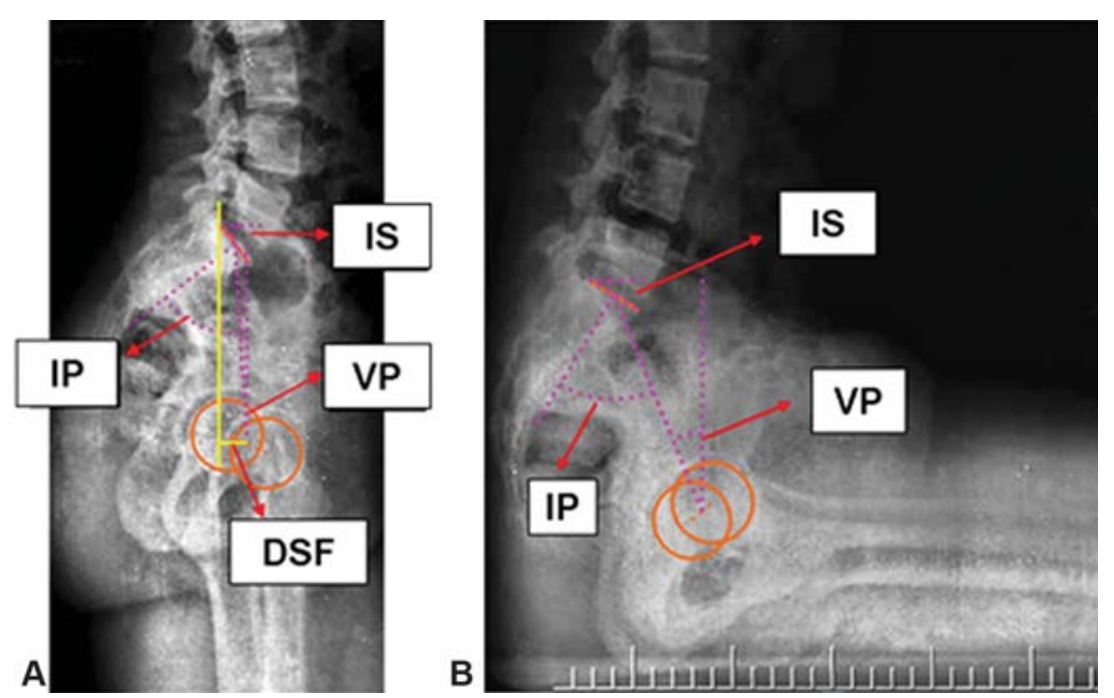

Fig. 3 Parâmetros radiográficos na incidência em perfil de coluna lombar-pelve-quadril-fêmur proximal. (A) Posição ortostática. (B) Posição sentada. Incidência Pélvica (IP); Versão pélvica (VP); Inclinação sacral (IS); Distância sacrofemoral (DSF).

associação positiva entre a presença da báscula da bacia com a $\mathrm{OA}$ de quadril foi observada antes da $\mathrm{ATQ}(\mathrm{RC}=4,6$; $\mathrm{IC}=1,3$ $15,4)$, mas não depois ( $R C=2,3 ; \mathrm{IC}=0,8-6,5)$. Os valores dos parâmetros radiográficos da coluna vertebral panorâmica estão detalhados na $\boldsymbol{- T a b e l a} \mathbf{1}$ e da coluna lombar-pelvequadril-fêmur proximal na - Tabela 2. Não foi observada diferença significativa na classificação do tipo de pelve e do tipo de CLP entre os pacientes antes e depois da ATQ e nem entre os pacientes e os controles.

\section{Discussão}

Na avaliação pré-operatória do paciente submetido à ATQ são realizadas radiografias de bacia AP com carga, incidência AP dos quadris e perfil do terço superior dos fêmures. Entretanto, não está prevista a avaliação dos parâmetros radiográficos do alinhamento sagital e espinopélvico, que são investigados em radiografias panorâmicas da coluna vertebral e na incidência em perfil de coluna lombar-pelve-quadril-fêmur proximal (em 
Tabela 1 Avaliação da coluna vertebral panorâmica

\begin{tabular}{|c|c|c|c|}
\hline \multirow[t]{2}{*}{ Variáveis } & \multirow{2}{*}{$\begin{array}{l}\text { Sem doença } \\
\text { articular }\end{array}$} & \multicolumn{2}{|l|}{ Osteoartrite } \\
\hline & & Antes ATQ & Depois ATQ \\
\hline $\begin{array}{l}\text { Alinhamento } \\
\text { Coronal } \\
\text { Global (mm) }\end{array}$ & $\begin{array}{l}3,1 \pm 9,3 \\
(-17,7-23,5)\end{array}$ & $\begin{array}{l}-1,07 \pm 10,9 \\
(-25,7-17,1)\end{array}$ & $\begin{array}{l}-1,08 \pm 17,9 \\
(-33,3-35,4)\end{array}$ \\
\hline $\begin{array}{l}\text { Báscula da } \\
\text { bacia }\left({ }^{\circ}\right)\end{array}$ & $\begin{array}{l}2,3 \pm 1,1 \\
(0,8-4,8)\end{array}$ & $\begin{array}{l}4,0 \pm 3,2^{\mathrm{a}} \\
(1,0-15,5)\end{array}$ & $\begin{array}{l}2,4 \pm 1,2^{b} \\
(0,9-4,9)\end{array}$ \\
\hline $\begin{array}{l}\text { Ângulo T1 } \\
\left.\text { pélvico ( }{ }^{\circ}\right)\end{array}$ & $\begin{array}{l}5,0 \pm 8,5 \\
(-14,2-21,8)\end{array}$ & $\begin{array}{l}6,9 \pm 9,0 \\
(-9,6-29,5)\end{array}$ & $\begin{array}{l}5,5 \pm 9,3 \\
(-8,5-35,3)\end{array}$ \\
\hline $\begin{array}{l}\text { Cifose } \\
\text { torácica }\left({ }^{\circ}\right)\end{array}$ & $\begin{array}{l}31,2 \pm 9,2 \\
(13,2-48,7)\end{array}$ & $\begin{array}{l}28,3 \pm 11,5 \\
(-4,4-47,0)\end{array}$ & $\begin{array}{l}29,8 \pm 11,7 \\
(5,3-47,0)\end{array}$ \\
\hline $\begin{array}{l}\text { Distância } \\
\text { sacrofemoral } \\
(\mathrm{mm})\end{array}$ & $\begin{array}{l}32,0 \pm 16,1 \\
(1,9-65,1)\end{array}$ & $\begin{array}{l}28,9 \pm 17,9 \\
(1,6-65,1)\end{array}$ & $\begin{array}{l}29,4 \pm 18,1 \\
(0,5-84,4)\end{array}$ \\
\hline $\begin{array}{l}\text { Eixo vertical } \\
\text { sagital }(\mathrm{mm})\end{array}$ & $\begin{array}{l}-9,8 \pm 35,9 \\
(-74,7-80,6)\end{array}$ & $\begin{array}{l}19,1 \pm 32,4^{\mathrm{a}} \\
(-40,9-82,3)\end{array}$ & $\begin{array}{l}5,1 \pm 36,0 \\
(-66,8-97,9)\end{array}$ \\
\hline $\begin{array}{l}\text { Inclinação T1 } \\
\text { espinopélvica } \\
\left({ }^{\circ}\right)\end{array}$ & $\begin{array}{l}-5,2 \pm 3,3 \\
(-11,4-3,0)\end{array}$ & $\begin{array}{l}-1,8 \pm 3,5^{a} \\
(-8,9-4,9)\end{array}$ & $\begin{array}{l}-3,1 \pm 3,8^{a} \\
(-9,6-6,7)\end{array}$ \\
\hline $\begin{array}{l}\text { Inclinação T9 } \\
\text { espinopélvica } \\
\left({ }^{\circ}\right)\end{array}$ & $\begin{array}{l}-10,1 \pm 3,7 \\
(-18,3--4,8)\end{array}$ & $\begin{array}{l}-5,8 \pm 4,9^{a} \\
(-13,7-3,6)\end{array}$ & $\begin{array}{l}-7,3 \pm 4,8^{a} \\
(-16,3-2,0)\end{array}$ \\
\hline $\begin{array}{l}\text { Lordose } \\
\text { lombar }\left({ }^{\circ}\right)\end{array}$ & $\begin{array}{l}59,8 \pm 9,7 \\
(42,9-85,2)\end{array}$ & $\begin{array}{l}53,1 \pm 26,4 \\
(-57,9-79,1)\end{array}$ & $\begin{array}{l}60,2 \pm 12,5 \\
(31,7-83,9)\end{array}$ \\
\hline $\begin{array}{l}\text { Razão de } \\
\text { C7/DSF }\end{array}$ & $\begin{array}{l}-1,7 \pm 4,8 \\
(-22,6-1,4)\end{array}$ & $\begin{array}{l}0,6 \pm 3,6^{a} \\
(-13,6-10,1)\end{array}$ & $\begin{array}{l}-0,6 \pm 6,2 \\
(-28,4-13,0)\end{array}$ \\
\hline $\begin{array}{l}\text { Discrepância } \\
\text { entre IP e LL }\end{array}$ & $\begin{array}{l}-10,7 \pm 10,4 \\
(-43,1-8,6)\end{array}$ & $\begin{array}{l}-6,8 \pm 13,5 \\
(-32,2-14,2)\end{array}$ & $\begin{array}{l}-9,7 \pm 13,2 \\
(-33,1-21,9)\end{array}$ \\
\hline
\end{tabular}

Abreviações: ATQ, artroplastia total de quadril; DSF, distância sacrofemoral; IP, incidência pélvica; LL, lordose lombar.

Valores expressos como média \pm desvio padrão (valores máximo-mínimo). a Significativamente diferente comparado ao grupo sem doença articular.

${ }^{\text {b}}$ Significativamente diferente comparado ao grupo antes da ATQ.

ortostase e sentado). Esta análise pode contribuir para esclarecer a relação entre as doenças degenerativas da coluna e do quadril. ${ }^{13}$ Considerando que a ATQ constitui uma das principais opções terapêuticas para os pacientes com OA de quadril, a avaliação dos parâmetros radiográficos pode ser uma ferramenta complementar no acompanhamento clínico do paciente, particularmente nos que permanecem sintomáticos após a cirurgia. $^{12}$

Em indivíduos com escoliose rígida, existe um risco aumentado de desequilíbrio da coluna vertebral após a ATQ decorrente da alteração abrupta da obliquidade pélvica. ${ }^{14}$ A permanência da curva escoliótica depois da ATQ pode ser explicada pela concomitância de alterações degenerativas, dificultando a compensação dos parâmetros de alinhamento espinopélvico e de anteversão pélvica. Assim, as deformidades da coluna que são estruturadas podem piorar após a ATQ. ${ }^{14}$

Recentemente, Esposito et al. ${ }^{15}$ observaram que $40 \%$ dos pacientes submetidos a ATQ primária apresentavam espondilodiscopatia degenerativa em diferentes níveis. Nossos resultados confirmam esta associação, uma vez que os parâmetros diagnósticos de doença degenerativa da coluna
Tabela 2 Avaliação da coluna lombar-pelve-quadril-fêmur proximal

\begin{tabular}{|c|c|c|c|}
\hline \multirow[t]{2}{*}{ Variáveis } & \multirow{2}{*}{$\begin{array}{l}\text { Sem doença } \\
\text { articular }\end{array}$} & \multicolumn{2}{|l|}{ Osteoartrite } \\
\hline & & Antes ATQ & Depois ATQ \\
\hline $\begin{array}{l}\text { Incidência } \\
\text { pélvica }\left({ }^{\circ}\right)\end{array}$ & $\begin{array}{l}50,1 \pm 9,5 \\
(32,3-70,4)\end{array}$ & $\begin{array}{l}50,4 \pm 10,1 \\
(34,6-75,6)\end{array}$ & $\begin{array}{l}50,4 \pm 10,1 \\
(34,6-75,6)\end{array}$ \\
\hline $\begin{array}{l}\text { Inclinação } \\
\text { sacral em } \\
\text { ortostática } \\
\left(^{\circ}\right)\end{array}$ & $\begin{array}{l}39,4 \pm 8,3 \\
(22,0-57,1)\end{array}$ & $\begin{array}{l}41,8 \pm 9,7 \\
(17,4-62,8)\end{array}$ & $\begin{array}{l}41,8 \pm 7,3 \\
(30,3-61,8)\end{array}$ \\
\hline $\begin{array}{l}\text { Inclinação } \\
\text { sacral } \\
\text { sentado }\left({ }^{\circ}\right)\end{array}$ & $\begin{array}{l}20,7 \pm 12,9 \\
(-6,9-49,2)\end{array}$ & $\begin{array}{l}8,9 \pm 7,8^{a} \\
(-10,1-25,2)\end{array}$ & $\begin{array}{l}11,7 \pm 9,6^{a} \\
(-13,9-28,8)\end{array}$ \\
\hline $\begin{array}{l}\text { Versão } \\
\text { pélvica em } \\
\text { ortostática } \\
\left({ }^{\circ}\right)\end{array}$ & $\begin{array}{l}10,7 \pm 8,0 \\
(-10,8-25,4)\end{array}$ & $\begin{array}{l}8,6 \pm 9,3 \\
(-7,4-36,0)\end{array}$ & $\begin{array}{l}8,6 \pm 9,9 \\
(-5,6-43,2)\end{array}$ \\
\hline $\begin{array}{l}\text { Versão } \\
\text { pélvica } \\
\text { sentado }\left({ }^{\circ}\right)\end{array}$ & $\begin{array}{l}29,4 \pm 13,1 \\
(4,2-68,6)\end{array}$ & $\begin{array}{l}41,5 \pm 10,4^{a} \\
(21,4-68,6)\end{array}$ & $\begin{array}{l}38,6 \pm 12,1^{\mathrm{a}} \\
(19,2-70,0)\end{array}$ \\
\hline
\end{tabular}

Abreviação: ATQ, artroplastia total de quadril.

Valores expressos como média \pm desvio padrão (valores máximo-mínimo). ${ }^{a}$ Significativamente diferente comparado ao grupo sem doença articular.

(osteófitos, redução do espaço intervertebral e artrose das articulações interapofisárias) foram prevalentes nos pacientes submetidos à ATQ. As alterações estruturais da coluna vertebral (alinhamento e altura dos corpos vertebrais) não foram associadas com a doença articular do quadril.

A assimetria entre as asas dos ilíacos (báscula da bacia), visualizada nas radiografias na incidência frontal em posição ortostática, pode ser secundária tanto à doença da coluna quanto à do quadril. ${ }^{16} \mathrm{~A}$ correção da báscula da bacia com a horizontalização da pelve e, consequentemente, da diferença de comprimento dos membros inferiores é um fator que contribui significativamente para o alívio da dor lombar no paciente com OA de quadril. ${ }^{14}$ Foi observada a redução do ângulo da báscula da bacia depois da ATQ para valores semelhantes aos valores dos voluntários sem doença articular, de acordo com o estudo de Abe et al., já que 61,5\% de um total de 195 pacientes submetidos à ATQ primária apresentaram correção do ângulo da báscula da bacia. ${ }^{14}$

O EVS e a razão de C7/DSF foram corrigidos depois da ATQ. A correção desses parâmetros após a cirurgia de ATQ sugere uma melhora significativa do equilíbrio da coluna vertebral no plano sagital. ${ }^{8}$

Quanto ao ângulo de IT1EP e IT9EP, nossos resultados também estão de acordo com os resultados de Weng et al. ${ }^{8}$ Foi observada a correção parcial das inclinações espinopélvicas, permitindo especular sobre o potencial da ATQ em promover a correção completa deste parâmetro ao longo do tempo. Entretanto, estudos com acompanhamentos mais longos seriam necessários para estabelecer a relação temporal entre ATQ e a normalização do ângulo da inclinação espinopélvica.

Apesar de não ter sido observada diferença entre os valores obtidos nos pacientes e nos voluntários sem doença articular para os parâmetros ATP, CT, DSF, LL, IP, discrepância 
entre IP e LL, IS e VP em ortostática, todas as medidas depois da ATQ foram alteradas e se aproximaram aos valores de referência. Destes parâmetros, foi demonstrado em estudos prévios $^{8,9}$ que a o ângulo da LL, como no presente estudo, não é influenciado pela ATQ.

Nossos resultados não mostraram relação entre ATQ e a normalização dos ângulos de IS e VP na posição sentada. Os resultados para ambos os parâmetros permaneceram muito próximos, antes e depois da ATQ. Em um estudo realizado com 89 pacientes submetidos à ATQ unilateral primária e 100 indivíduos sem doença no quadril, embora tenha sido observada redução da amplitude do movimento pélvico e diminuição da retroversão pélvica nos pacientes submetidos a ATQ, como no presente estudo, não houve normalização dos parâmetros. Entretanto, os autores ressaltam a importância em avaliar este parâmetro uma vez que ele condiciona a redução na anteversão acetabular, o que pode predispor a luxação posterior da prótese. ${ }^{2}$

A diferença da VP na mudança da posição ortostática para sentada indica o tipo de CLP. O arco de movimento sagital entre o tronco e as coxas é dividido entre o CLP e os quadris nas atividades diárias. Os pacientes com doença degenerativa da coluna vertebral podem evoluir com desequilíbrio do CLP devido à rigidez da coluna e consequente sobrecarga compensatória no quadril. ${ }^{10}$ No presente estudo, após a ATQ todos os pacientes apresentaram diferença.$>10^{\circ}$ entre a versão pélvica ortostática e sentada, indicando um CLP flexível, que pode estar associado com baixo risco de impacto da prótese. ${ }^{10}$

A principal limitação do estudo foi tamanho amostral e a ausência das informações do Harris Hip Score para correlacionar os achados de imagem com queixas de dor, função, deformidade e mobilidade do quadril.

\section{Conclusão}

A deformidade da coluna pode ser compensatória às alterações do quadril, uma vez que o ângulo da báscula da bacia, o EVS e a razão C7/DSF foram corrigidos após a ATQ. Esta conclusão pode ajudar o cirurgião na tomada de decisão quanto ao posicionamento dos componentes da ATQ nos pacientes com doença concomitante na coluna vertebral e na articulação coxofemoral.

Conflito de interesses

Os autores declaram não haver conflito de interesses.

\section{Referências}

1 Yoshimoto H, Sato S, Masuda T, et al. Spinopelvic alignment in patients with osteoarthrosis of the hip: a radiographic comparison to patients with low back pain. Spine 2005;30(14):1650-1657

2 Sariali E, Lazennec JY, Khiami F, Gorin M, Catonne Y. Modification of pelvic orientation after total hip replacement in primary osteoarthritis. Hip Int 2009;19(03):257-263

3 Watelain E, Dujardin F, Babier F, Dubois D, Allard P. Pelvic and lower limb compensatory actions of subjects in an early stage of hip osteoarthritis. Arch Phys Med Rehabil 2001;82(12):1705-1711

4 Fellet A, Fellet AJF. Osteoartrose: uma revisão. Rev Bras Med 2007; 64:55-61

5 Redmond JM, Gupta A, Nasser R, Domb BG. The hip-spine connection: understanding its importance in the treatment of hip pathology. Orthopedics 2015;38(01):49-55

6 Blizzard DJ, Sheets CZ, Seyler TM, et al. The Impact of Lumbar Spine Disease and Deformity on Total Hip Arthroplasty Outcomes. Orthopedics 2017;40(03):e520-e525

7 Blizzard DJ, Nickel BT, Seyler TM, Bolognesi MP. The Impact of Lumbar Spine Disease and Deformity on Total Hip Arthroplasty Outcomes. Orthop Clin North Am 2016;47(01):19-28

8 Weng W, Wu H, Wu M, Zhu Y, Qiu Y, Wang W. The effect of total hip arthroplasty on sagittal spinal-pelvic-leg alignment and low back pain in patients with severe hip osteoarthritis. Eur Spine J 2016;25(11):3608-3614

9 Ben-Galim P, Ben-Galim T, Rand N, et al. Hip-spine syndrome: the effect of total hip replacement surgery on low back pain in severe osteoarthritis of the hip. Spine 2007;32(19):2099-2102

10 Rivière C, Hardijzer A, Lazennec JY, Beaulé P, Muirhead-Allwood S, Cobb J. Spine-hip relations add understandings to the pathophysiology of femoro-acetabular impingement: A systematic review. Orthop Traumatol Surg Res 2017;103(04):549-557

11 Rivière C, Lazennec JY, Van Der Straeten C, Auvinet E, Cobb J, Muirhead-Allwood S. The influence of spine-hip relations on total hip replacement: A systematic review. Orthop Traumatol Surg Res 2017;103(04):559-568

12 Phan D, Bederman SS, Schwarzkopf R. The influence of sagittal spinal deformity on anteversion of the acetabular component in total hip arthroplasty. Bone Joint J 2015;97-B(08):1017-1023

13 Lazennec JY, Brusson A, Rousseau MA. Hip-spine relations and sagittal balance clinical consequences. Eur Spine J 2011;20 (Suppl 5):686-698

14 Abe Y, Sato S, Abe S, Masuda T, Yamada K. The impact of the leglengthening total hip arthroplasty on the coronal alignment of the spine. Scoliosis 2015;10(Suppl 2):S4

15 Esposito CI, Miller TT, Kim HJ, et al. Does Degenerative Lumbar Spine Disease Influence Femoroacetabular Flexion in Patients Undergoing Total Hip Arthroplasty? Clin Orthop Relat Res 2016; 474(08):1788-1797

16 Ormond Filho AG, Gonzalez MT, Homsi C, Stump XMG. Biomecânica e alterações de alinhamento do eixo pelvirraquidiano. In: Fernandes JL, Maciel Junior F, Muller CIS, Giuseppe D, Rocha AJ, editores. Coluna Vertebral. Série Colégio Brasileiro de Radiologia e Diagnóstico por Imagem. Rio de Janeiro: Elsevier Editora Ltda; 2013:77-106 